\title{
Avaliação da conectividade hidrológica da bacia do rio Corumbataí, SP.
}

\author{
Angélica Nardo Caseri \\ Silvio Frosini de Barros Ferraz \\ Felipe Rossetti de Paula \\ Universidade Estadual Paulista - UNESP \\ Rua 13B, 437, Bela Vista - 13506-748 - Rio Claro - SP, Brasil \\ angelika_caseri@hotmail.com
}

\begin{abstract}
This work, in the first time, aim to the construction of a cartographic base, the Corumbataí river basin, 1:50000 evidencing the hydrologic topology, which will count rivers'segments, areas of contribution and catchments, these last ones causing the reduction of the connectivity. After, the flow direction, the hydrologics distances of random points and the level of catchments were calculated. By means of the cartographic base, and of hydrologic studies, as, the evaluation of the connectivity hydrologic, the construction of the contribution areas, and the calculation of the favorable flow and distances inside the drainage, was possible to accomplishment metric space analyses incorporating, as distance between points inside the drainage, the level of catchments inside each stretch, and, finally, the construction of the connectivity tables, that will facilitate the agreement with the connections between the basin's analyzed points. The classification of the stretches will be carried through means of different analyses, being that the results will be useful for the establishment of strategies of the aquatic biodiversity conservation in the region.
\end{abstract}

Palavras chaves: conectividade hidrológica, bacia do rio Corumbataí, microbacias. 


\section{Introdução}

Os ecossistemas naturais são caracterizados por apresentarem uma estrutura (formada pelo meio biótico e meio abiótico) e uma função, sendo esta representada pelas relações entre os seres vivos e o ambiente físico local (Chapin III et al., 2002). Estas relações entre os elementos de um ecossistema, como os fluxos de energia, materiais e nutrientes, são afetadas por diversos fatores, dentre eles a conectividade existente dentro de um ecossistema ou entre ecossistemas distintos (Crooks, 2006).

A conectividade é um fator marcante dentro de uma rede de drenagem (sistema composto por ecossistemas lóticos de rios e riachos) devido ao fluxo unidirecional da água no interior da rede. Este fluxo unidirecional exerce forte influência nas características físicas e nos processos ecológicos dos ecossistemas lóticos, sendo fundamental para a manutenção da sua estrutura e do seu funcionamento (Pringe, 2006).

Este projeto tem como área de estudo a bacia do rio Corumbataí, a qual se localiza na região centro-leste do estado de São Paulo (Projeto Corumbataí, 2001), possuindo grandes influências de ações antrópicas, principalmente pela atividade agrícola, urbanização e extração de areia (Bacci, 1994).

A partir do intuito de se analisar o nível de fragmentação da bacia do rio Corumbataí, foi construída uma base cartográfica da região, para possibilitar a realização de estudos espaciais adicionados a índices métricos entre pontos estabelecidos dentro da rede de drenagem, a contribuição de descarga que um local ocasiona para o outro, a quantificação de açudes ao decorrer das microbacias e estabelecer o nível de fragmentação no ciclo hidrológico.

A análise de distâncias na rede e a quantificação da quebra da conectividade de uma rede de drenagem subsidia estudos posteriores relacionados à reconstrução da região afetada por meio da restauração das áreas degradadas, e a conservação dos trechos que não sofreram danos ocasionados pela atividade humana (Salati, 1996). A restauração das regiões afetadas pelas atividades humanas requer a integração de ações que envolvem a bacia hidrológica, o ecossistema lótico e seus componentes (físicos, químicos e bióticos), possibilitando o diagnóstico da degradação, permitindo a avaliação das alternativas para a recuperação e custos da mesma.

\section{Objetivos}

Como objetivo principal caracteriza-se a avaliação da conectividade hidrológica da bacia do rio Corumbataí.

Como objetivos secundários pode-se citar: a construção de uma rede hidrográfica topologicamente ajustada na escala 1:50.000 e a classificação dos trechos da rede hidrográfica quanto a sua conectividade como forma de subsídio para planejamento da conservação. 


\section{Material e Métodos}

\section{1. Área de estudo}

A região abordada é a bacia do rio Corumbataí (o rio Corumbataí nasce na localização geográfica, longitude $47^{\circ} 22^{\prime} 33$ oeste e latitude $22^{\circ} 10^{\prime} 05$ sul), sendo esta bacia, uma sub-bacia do Rio Piracicaba, centro-oeste do estado de São Paulo (Deplan, 2001).

A altitude da região varia de 470 metros, na foz do rio Corumbataí, a 1058 metros, na Serra do Cuscuzeiro em Analândia (SP). Ela ocupa uma área aproximada de $1700 \mathrm{~km}^{2}$, que abrange totalmente o município de Rio Claro e, parcialmente, os municípios de Analândia, Corumbataí, Itirapina, Charqueada, Ipeúna, Santa Gertrudes e Piracicaba (Koffer, 1994), possuindo uma rede hidrográfica com padrão dendrítico (Viadanna, 1985).

O rio Corumbataí, um dos principais afluentes, no seu alto curso, é caracterizado por fluir vales estreitos, encachoeirados com pequenas quedas e corredeiras, o que lhe da maior velocidade e um fluxo constante na região. Seu curso médio possui, baixa declividade, tornando-se um rio mais caudaloso e lento, e, por fim, no seu baixo curso, o rio Corumbataí apresenta um canal sinuoso e retilíneo com vales abertos o que lhe permite retornar a sua característica inicial de maior velocidade (Garcia, 2006).

A bacia do rio Corumbataí está localizada em uma porção do território paulista de grande importância econômica, sob o ponto de vista do desenvolvimento agrícola e industrial (Valente 2001). A atividade agrícola temporária predominante é de cana-deaçúcar e nas lavouras permanentes, o café e a laranja ocupam lugar de destaque (Garcia, 2006). Também deve-se considerar o grande potencial de mineração da região, devido a existência de substâncias utilizadas para a fabricação de cerâmicas, corretivos de solo e revestimentos cerâmicos (Colturato, 2002). Constata-se que 40,75\% da área é ocupada por pastagens, $26,74 \%$ pela plantação de cana-de-açúcar, a mineração, a cultura anual e a fruticultura ocupam respectivamente $0,13 \%, 2,34 \%$ e $0,82 \%$, apenas $9,57 \%$ é ocupado por floresta nativa (Vettorazzi, 2000). Por meio destas ações citadas, a malha viária da região possui alto crescimento gerando a construção de represamentos ao longo da bacia. Estes, por sua vez, propiciam a quebra da conectividade hidrológica (Pringle, 2006).

\subsection{Construção da base cartográfica}

Para se obter os dados necessários para a caracterização e construção da uma base cartográfica representando a hidrologia da bacia do rio Corumbataí, em formato vetorial, foram utilizadas cartas topográficas de 1:50000 (IBGE, 1979) de cada região que a bacia se encontra, sendo estas localizadas nas cidades de Araras, Corumbataí, Limeira, Piracicaba, Rio Claro, São Carlos, São Pedro e Leme ( Projeto Piracena, 2000).

A partir das cartas topográficas, a rede de drenagem digital foi reformulada no software ArcGis9.2, procurando-se corrigir inconsistências. Para a reconstrução da hidrografia, foi feita a correção de trajetos de rios e de represamentos, a conexão de todos segmentos e corpos d'água e o ajuste das junções, criando-se uma base de dados topologicamente consistente no formato Geodatabase. 


\subsection{Identificação dos segmentos e construção das áreas de contribuição}

\subsubsection{Individualização de segmentos}

A partir da construção da base cartográfica, todos os segmentos, correspondentes a riachos, ribeirões e rios que compõem a rede, foram individualizados e identificados. Também foram identificados elementos que representem quebras de conectividade da rede, como os represamentos e quebras ocasionadas pela malha viária. Para esta última atividade, foramutilizadas imagens aéreas georreferenciadas (escala 1:30.000) para identificação das interrupções na rede hidrográfica

Por meio das extensões Network Analyst (ESRI, 2006), foi validada a conectividade de cada segmento, sendo que nesta etapa é possível a identificação de trechos não interligados topologicamente, possibilitando a correção final da rede hidrográfica.

\subsubsection{Avaliação da conectividade hidrológica}

Por meio da conversão do banco de dados gerados para o modelo Geodatabase, foi utilizada a extensão FloWS - Functional Linkage of Water basins and Streams (Theobald et al, 2006) para realizar análises espaciais incorporando métricas e índices, como distância jusante-montante entre pontos dentro da rede, a construção das áreas de contribuição de cada segmento, o cálculo do acumulo de água em um trecho selecionado e o calculo do nível de fragmentação dentro de uma rede de drenagem, devido aos represamentos e a malha viária que propiciam a quebra da conectividade hidrológica.

\subsection{3. Áreas de contribuição}

Posteriormente a construção da base cartográfica,foi criada a área de contribuição para cada trecho único que compõe a rede, sendo que esta área representa um sub-componente de uma microbacia que drena diretamente em um dado segmento de rio.

Para o cálculo destas áreas deverão ser utilizados a rede hidrográfica gerada e o modelo digital do terreno da bacia. Como resultado foi obtido um arquivo vetorial de polígonos que representem as áreas de contribuição de cada segmento de rio delimitadas (RCA - Reach Catchment Áreas) para a bacia do Rio Corumbataí. Estes procedimentos foram realizados através da utilização da extensão Flows (Functional Linkage of Water basins and Streams). 


\section{Resultados e Discussão}

Por meio dos resultados obtidos do cálculo das distâncias hidrológicas entre as microbacias selecionadas, pode-se observar que alguns trechos apresentam grandes distâncias, como é o caso das microbacias 20 e 652, com $77 \mathrm{Km}$. Entretanto, em pequena quantidade, também foram encontrados pequenos trechos, como por exemplo entre as microbacias 47 e 59, com $1,1 \mathrm{Km}$, sendo esta caracterizada por possuir menor distância. Desta forma, é possível que as microbacias que possuem menor distância possam contribuir, em uma taxa mais elevada, com microorganismos e organismos para os seus ecossistemas.

Em relação aos represamentos presentes nos trechos que ligam as microbacias, pode-se observar que todas possuem algum tipo de quebra hidrológica, em destaque encontram-se as microbacias 2, 8 e 20, que em suas distâncias apresentam, como valor máximo, 7 represamentos entre cada ligação estabelecida com as microbacias 77,83 , 137, 174, 188 e 308. Devido a estes represamentos é possível que existam alterações na estrutura das comunidades presentes, como, interrupções nas rotas migratórias; o desaparecimento de espécies e a diminuição dos estoques das mesmas (FERREIRA e CARAMASCHI, 2005). Também é provável que ocorra alterações nas condições bióticas e abióticas devido à deposição de sedimentos, a decomposição da vegetação e a destruição da mata ciliar nas áreas represadas (BRITSKI, 1994). Deste modo, por meio destes fatos, provavelmente os represamentos afetam com maior intensidade a biologia das espécies nativas, sendo enfatizadas as espécies de peixes migratórias (CARVALHO et al., 1998a e b).

Por meio dos resultados obtidos até o momento, não se pode afirmar pontos que possuam maior ou menor conectividade hidrológica. Entretanto, possivelmente os trechos que podem possuir maiores dificultados de se interligarem, são identificados pelas microbacias 2, 8 e 20, levando-se em consideração os represamentos existentes, e as microbacias 635 e 652, em relação as distâncias hidrológicas.

Desta maneira, pode-se mencionar que os represamentos e as distâncias hidrológicas podem ocasionar transformações nos meios bióticos e abióticas, evidenciando o meio biótico devido às espécies migratórias. 


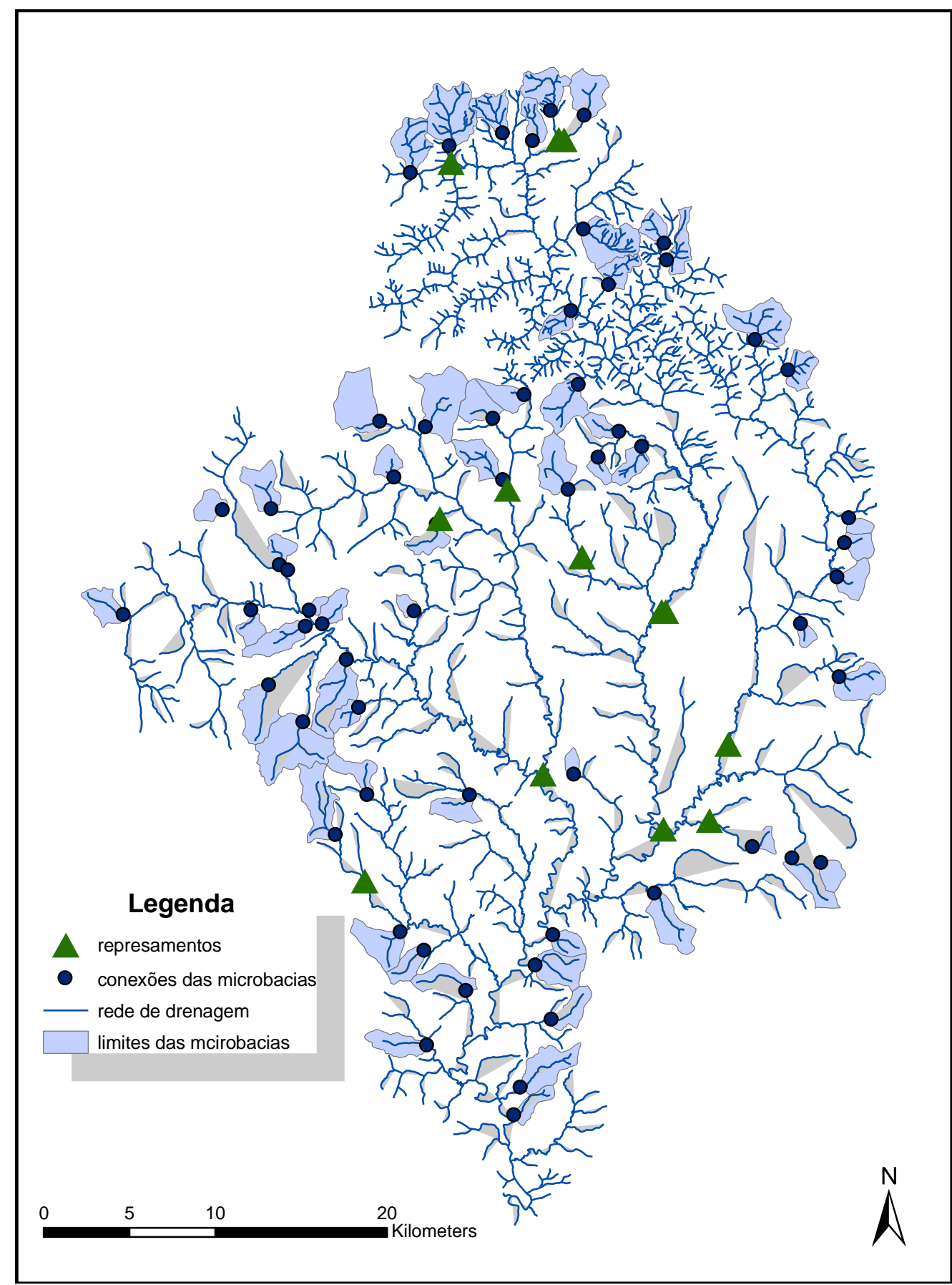

Figura 1. Apresentação da rede de drenagem, das 60 microbacias analisadas ( utilizadas para o cálculo das distâncias hidrológicas), e dos represamentos identificados. 


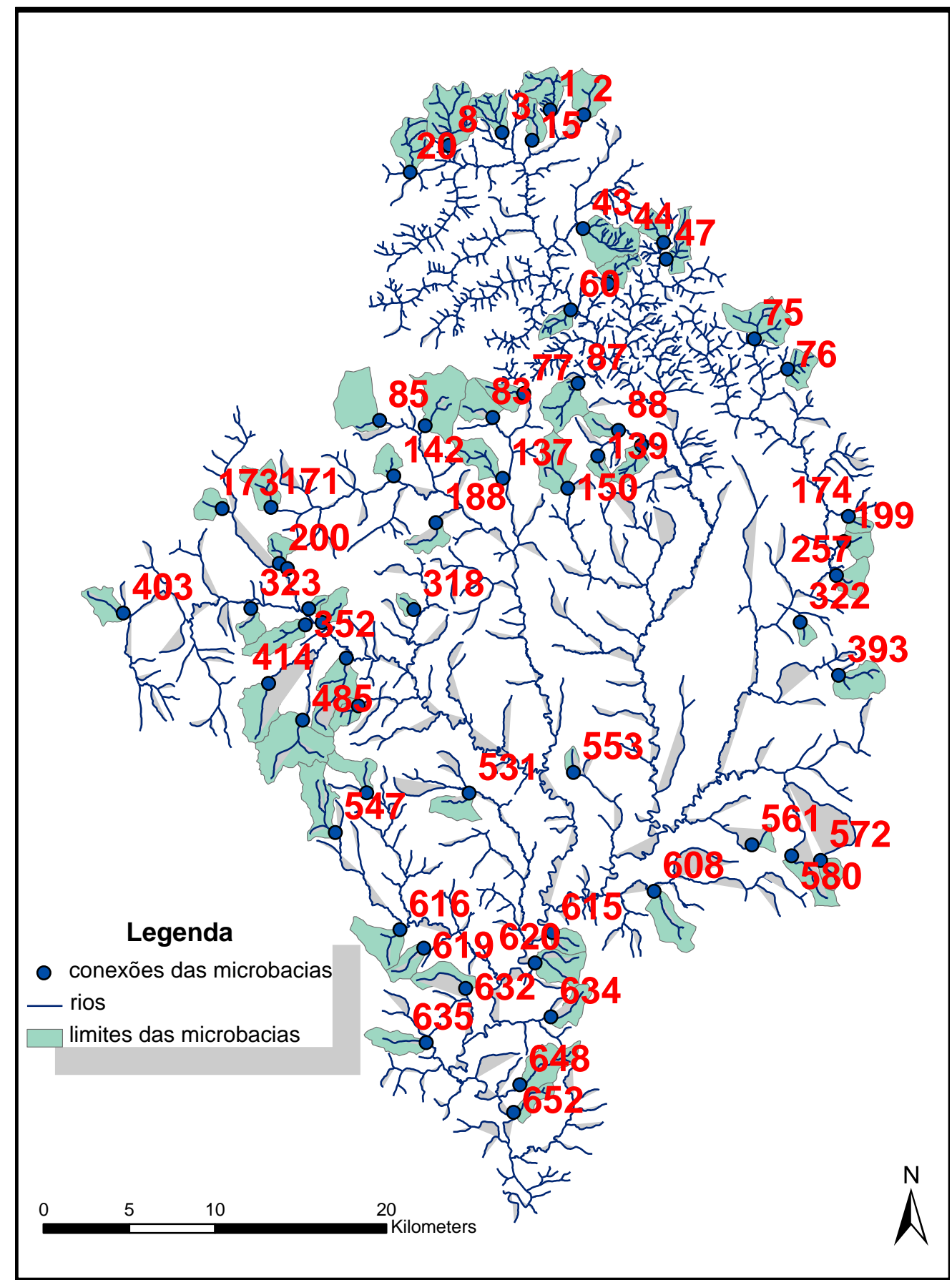

Figura 2. Identificação das 60 microbacias estudadas para a análise da quantificação da conectividade hidrológica dentro da bacia do rio Corumbataí. 


\section{Referências bibliográficas}

Bacci, D.L.C. Extração de Areia na Bacia do Rio Corumbataí.115.Tese (Mestrado na área de concentração de geociências e meio ambiente) - Instituto de Geociências e Ciências Exatas, Universidade Estadual Paulista, Rio Claro, 1994.

BRITSKI, H. A. A fauna de peixes brasileiros de água doce e o represamento de rios. In: COMASE: Seminário sobre fauna aquática e o setor elétrico. Rio de Janeiro. p. 2330, 1994.

CARVALHO, E. D.; SILVA, V. F. B.; FUJIHARA, Y. C.; HENRY, R.; FORESTI, F. Diversity of fish species in the River Paranapanema - Jurumirim Reservoir transition region (São Paulo, Brazil). Italian Journal of Zoology, n. 65, Suppl.: p.325-330, 1998a.

CARVALHO, E. D.; FUJIHARA, C. Y.; HENRY, R. Study on the ichthyofauna of the Jurumirim reservoir (Paranapanema river, São Paulo State, Brazil): fish production and dominant species in three sites. Verh. Internat. Verein. Limnol, n. 26, p. 2199-2202, 1998 b.

IBGE- Fundação Instituto Brasileiro de Geografia e Estatística - Escala 1:50.000. Proj. Universal Transversa de Mercator. 1979.

FERREIRA, M. F. N. e CARAMASCHI, E. P. Aspectos da estratégia reprodutiva de machos de Teleósteos na área de influência da Usina Hidrelétrica Serra da Mesa, Alto rio Tocantins, GO. In: NOGUEIRA, M. G.; HENRY, R.; JORCIN, A. (Eds.): Ecologia de reservatórios: impactos potenciais, ações de manejo e sistemas em cascata. São Carlos: Editora RiMa. p. 305-328, 2005.

PROJETO CORUMBATAÍ. Plano Diretor:Conservação dos recursos hídricos por meio da recuperação e da conservação da cobertura florestal da bacia do rio Corumbataí. Relatório Técnico do IPEF, Piracicaba, 2001, 301p.

Theobald, D.M., Norman, J.B.; Peterson, E.; Ferraz, S.F.B.; Wade,A.; Sherburne, M.R. Functional Linkage of Water basins and Streams ( FloWS) v1 User's Guide: ArcGIS tools for Network-based analysis of freshwater ecosystems. Natural Resource Ecology Lab, Colorado State University, Fort Collins, CO. 2006.43p.

Chapin III,F.S.; Matson, P.A.; Mooney,H.A. Principles of Terrestrial Ecosystem Ecology.1.ed. New York: Springer, 2002. 436p.

Crooks, K.R.Connectivity Conservationa: Maintaining Connections for Nature. In: Crooks, K.R. Sanjayan, M. Connectivity Conservation.1.ed. New York: Cambridge University Press, 2006. 712p.

Pringe, C. Hydrologic Connectivity: A Neglected Dimension of Conservation Biology. In: Crooks, K.R. Sanjayan, M. Connectivity Conservation.1.ed. New York: Cambridge University Press, 2006. 712p.

SALATI, E. Análise ambiental sintética e qualidade da água do rio Corumbataí (SP) como subsídio para planejamento regional integrado da bacia de drenagem do rio Corumbataí. São Carlos, 1996. 198p. Tese (Doutorado) - Ciências da Engenharia Ambiental, Universidade Federal de São Carlos.

VETTORAZZI, C.A.; VALENTE, R.O.A.; BALLESTER, M.V.R. Forest fire hazard mapping in a GIS environment for a river basin in the State of São Paulo, Brazil. In: 
International Coference on Geospatial Information in Agriculture and Forestry, 2., Lake Buena Vista, 2000. Procedings. Ann Arbor: ERIM International, 2000. v. 1, p. $10-12$.

ESRI. ArcGis Network Analyst Tutorial. United States of American: Copyright, 2006. $34 \mathrm{p}$.

PROJETO PIRACENA. Banco de dados "Geoprocessamento". http://www.cena.usp.br/piracena. 2000. 